\title{
TITLE:
}

\section{Abnormal hyperintensity in cerebellar efferent pathways on diffusion-weighted imaging in a patient with heat stroke.}

\author{
AUTHOR(S): \\ Fushimi, Y; Taki, H; Kawai, H; Togashi, K
}

\section{CITATION:}

Fushimi, Y...[et al]. Abnormal hyperintensity in cerebellar efferent pathways on diffusionweighted imaging in a patient with heat stroke.. Clinical radiology 2012, 67(4): 389-392

\section{ISSUE DATE:}

2012-04

URL:

http://hdl.handle.net/2433/179453

\section{RIGHT:}

(C) 2013 Elsevier B.V.; This is not the published version. Please cite only the published version.; この論文は出版社版でありません。引用の際に は出版社版をご確認ご利用ください。 
Title

Abnormal hyperintensity in cerebellar efferent pathwsays on diffusion-weighted imaging in a patient with heat stroke 


\section{Text}

\section{Introduction}

Heat stroke is a potentially critical condition characterized by hyperthermia and accompanied by various complications such as multi-organ failure and disseminated intravascular coagulation (DIC). Heat stroke can also cause various neurological dysfunctions, most commonly due to involvement of the cerebellum (1). Lee et al. reported possible selective vulnerability of cerebellar neurons to heat injury (2).

Previous case reports concerning MRI findings of heat stroke have noted cerebellar findings such as diffuse atrophy of the cerebellum in the chronic phase (1), and hyperintense cerebellar cortices (3). Diffusion-weighted imaging (DWI) is useful for detecting hyperintense dentate nuclei (4), hyperintensity of bilateral superior cerebellar peduncles (SCPs), the central tegmentum of the midbrain (decussation of SCPs), and thalami (5), and the central tegmentum of the midbrain (6). Hyperintense lesions in the hippocampi, cerebellum, and cerebral cortices (7), and lesions of the splenium have also been reported (4). We report herein a case of heat stroke presenting with hyperintense bilateral dentate nuclei, SCPs, decussation of SCPs on DWI and mild hyperintense cerebral cortices on DWI. 


\section{Case report}

A 53-year-old woman was emergently transferred to our hospital with impaired consciousness and high body temperature on a summer day when the peak temperature was $38^{\circ} \mathrm{C}$. She was found lying outside her house in the afternoon, and had been in good health in the morning. She was in a deep coma and rectal temperature was $40^{\circ} \mathrm{C}$. Laboratory examinations showed hepatic dysfunction and disseminated intravascular coagulation, so severe heat stroke was diagnosed. MRI on admission (including DWI) showed no abnormalities (Fig. 1). Aggressive external cooling and treatment for DIC were started immediately and appeared effective, but she remained comatose. DWI on hospital day 9 showed abnormal signals hyperintensities in bilateral dentate nuclei, SCPs, and central tegmentum of the midbrain (decussation of SCPs) (Fig. 2). Apparent diffusion coefficients (ADCs) in these regions showed intermediate values (Fig. 2). T2-weighted imaging was of poor quality due to motion artifacts, however, mild hyperintensities in bilateral dentate nuclei were visualized. Intermediate values for ADC indicated some extent of cytotoxic edema and vasogenic edema. DWI also showed mild signal hyperintensities with high ADC in the cerebral cortices, indicating vasogenic edema (Fig. 2).

The patient gradually recovered consciousness, but severe cerebellar 
dysfunction remained. MRI obtained 2 months after admission revealed cerebellar and cerebral atrophy (Fig. 3). DWI showed no abnormal intensity in the cerebellum or midbrain. She was transferred to a rehabilitation facility 3 months after admission.

\section{Discussion}

The cerebellum is vulnerable to heat injury. Loss of Purkinje cells residing in the cerebellar cortex due to hyperthermia has been reported in neuroleptic malignant syndrome (2). Bazille et al. reported almost total loss of Purkinje cells in all of three patients with heat stroke in a postmortem study (6). Increased expression of heat shock protein 70 (HSP70) was noted near remaining Purkinje cells and adjacent Bergmann glia, suggesting selective vulnerability of Purkinje cells to heat injury (6).

The cerebellar efferent pathway enters the tegmentum of the midbrain through the SCP, and divides into two tracts: the cerebellorubral tract ending at the red nucleus; and the cerebellothalamic tract ending at the venterolateral thalamic nucleus traversing the red nucleus. The cerebellar efferent pathway shows myelin pallor in the case of heat stroke, and nerve cell degeneration has also been observed in the dentate nucleus and ventrolateral nucleus of the thalamus. DNA breakage of neurons in the dentate nucleus and ventrolateral nucleus as well as Purkinje cell axon degeneration indicate that this 
neuronal damage might have derived from deafferentation (6).

MRI findings associated with cerebellar efferent pathways have been reported as follows: bilateral dentate nuclei on hospital day 4 (4), bilateral superior cerebellar peduncles and thalami on DWI on hospital day 7 (5), and decussation of SCPs on hospital day 12 (6). Despite extensive involvement of cerebellar efferent pathways in our patient, DWI obtained immediately after transfer showed no abnormalities. These findings might support the idea that abnormal signals only appear in cerebellar efferent pathways over time, potentially consistent with the mechanisms of deafferentation. A postmortem study already showed axonal swelling of the cerebellar efferent pathway by 1 day after heat injury in one case (6), so the interval required for the deafferentation effect appears to depend on the extent of heat injury. DWI findings several days after heat injury may provide additional information regarding the prognosis of heat stroke, even if no abnormality is apparent on admission.

Multiple cerebral cortices are involved in patients with heat stroke, as in our case, but the mechanisms have not been well discussed. Most cerebral lesions in this case were not continuous or symmetrical, and no signs suggested involvement along the thalamocortical tract. A previous report speculated on the potential presence of diffuse cerebral edema due to vasogenic edema on computed tomography performed on the day 
of admission (3), but our case showed no abnormalities suggestive of brain edema on initial MRI. A postmortem study in another case showed no cerebral lesions suggesting central nervous system (CNS) changes with heat stroke, but found nonspecific slight diffuse astrocytosis (6). Differential diagnosis of cerebral cortical lesions in our case might include other CNS abnormal conditions such as postictal encephalopathy, encephalitis, posterior reversible encephalopathy syndrome. However, no associated clinical symptoms were observed.

Conclusion

In summary, we present an interesting case of heat stroke with abnormal hyperintensity in cerebellar efferent pathways on DWI. MRI findings of heat stroke may change over time, even if no abnormalities are apparent at onset. DWI findings several days after heat injury may add some information regarding the prognosis of heat stroke, even if no abnormality is seen on admission. 


\section{References}

1. Albukrek D, Bakon M, Moran DS, Faibel M, Epstein Y. Heat-stroke-induced cerebellar atrophy: clinical course, CT and MRI findings. Neuroradiology. 1997;39(3):195-197.

2. Lee S, Merriam A, Kim TS, Liebling M, Dickson DW, Moore GR. Cerebellar degeneration in neuroleptic malignant syndrome: neuropathologic findings and review of the literature concerning heat-related nervous system injury. J Neurol Neurosurg Psychiatry. 1989;52(3):387-391.

3. Mahajan S, Schucany WG. Symmetric bilateral caudate, hippocampal, cerebellar, and subcortical white matter MRI abnormalities in an adult patient with heat stroke. Proc (Bayl Univ Med Cent). 2008;21(4):433-436.

4. Lee JS, Choi JC, Kang SY, Kang JH, Park JK. Heat stroke: increased signal intensity in the bilateral cerebellar dentate nuclei and splenium on diffusion-weighted MR imaging. AJNR Am J Neuroradiol. 2009;30(4):E58.

5. Ookura R, Shiro Y, Takai T, Okamoto M, Ogata M. Diffusion`weighted magnetic resonance imaging of a severe heat stroke patient complicated with severe cerebellar ataxia.

Intern Med. 2009;48(12):1105-1108.

6. Bazille C, Megarbane B, Bensimhon D, et al. Brain damage after heat stroke. J 
Neuropathol Exp Neurol. 2005;64(11):970-975.

7. Sudhakar PJ, Al-Hashimi H. Bilateral hippocampal hyperintensities: a new

finding in MR imaging of heat stroke. Pediatr Radiol. 2007;37(12):1289-1291. 


\section{Figure captions}

Fig. 1

DWI on admission shows no abnormalities in bilateral dentate nuclei (a), SCPs (b), or central tegmentum of the midbrain (decussation of SCPs) (c) (arrows). ADC in bilateral dentate nuclei shows intermediate values (d, arrows). T2-weighted imaging shows normal appearance of dentate nuclei (e, arrows). Note that no cerebellar atrophy is apparent. DWI also shows normal appearance of cerebral cortices (f).

\section{Fig. 2}

DWI on hospital day 9 showing abnormal hyperintensities in bilateral dentate nuclei (a), SCPs (b), or central tegmentum of the midbrain (decussation of SCPs) (c) (arrows). ADC in bilateral dentate nuclei shows intermediate values (d, arrows). T2-weighted imaging was of poor quality due to motion artifacts, however, mild hyperintensities in bilateral dentate nuclei were visualized (e, arrows). DWI also shows mild hyperintesities (f, arrowheads) with high ADC in cerebral cortices.

\section{Fig. 3}


T2-weighted imaging obtained 2 months after admission reveals cerebellar (a) and cerebral atrophy (b). 
Fig. 1

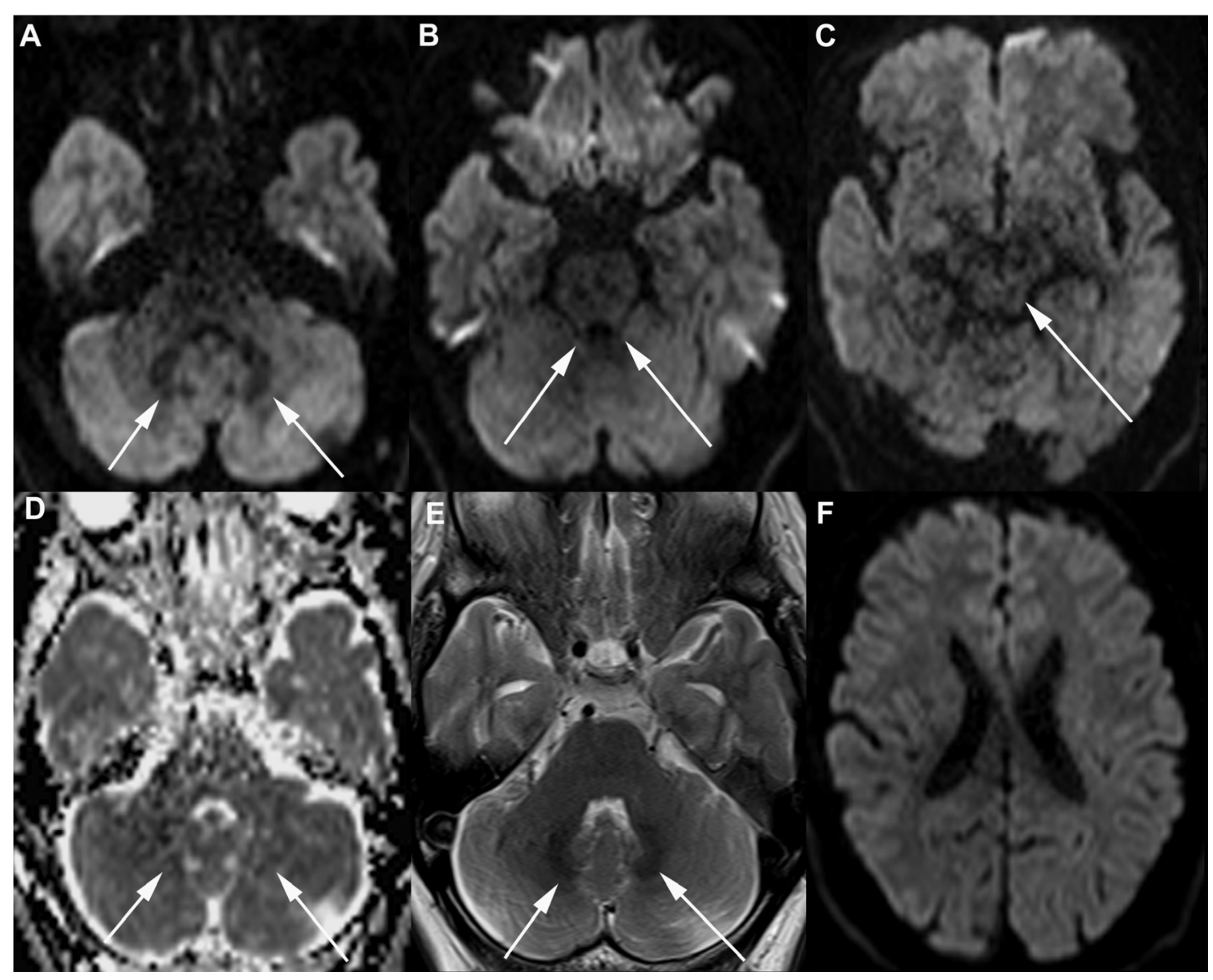






Fig. 2

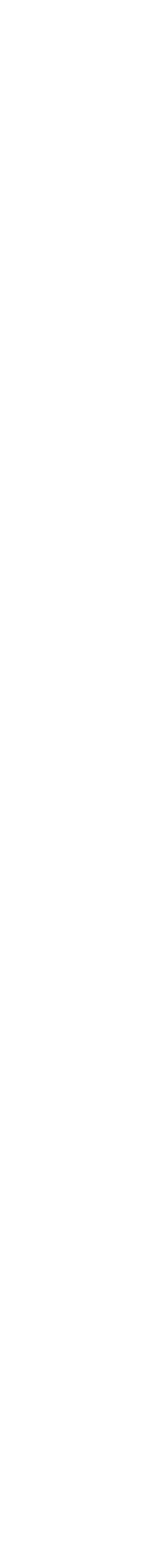

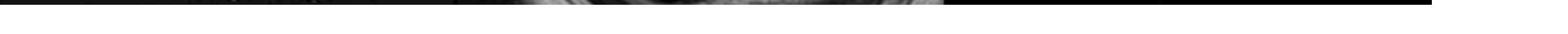
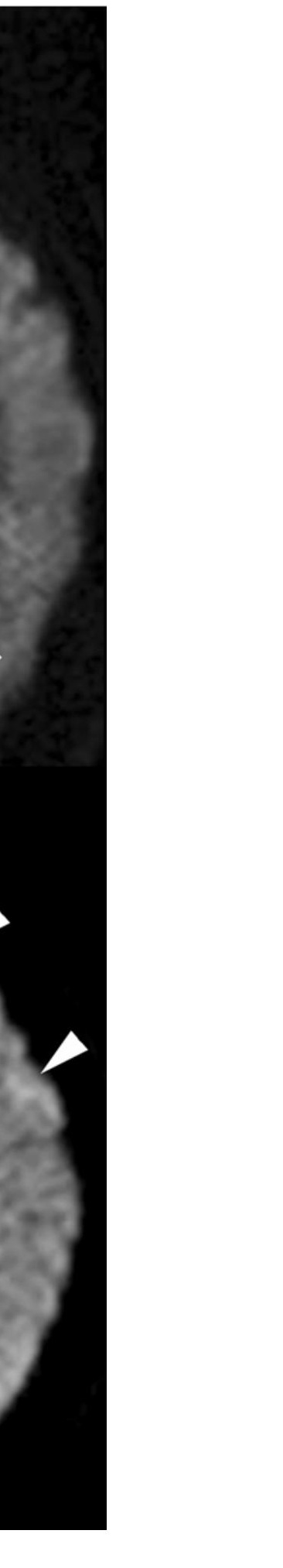


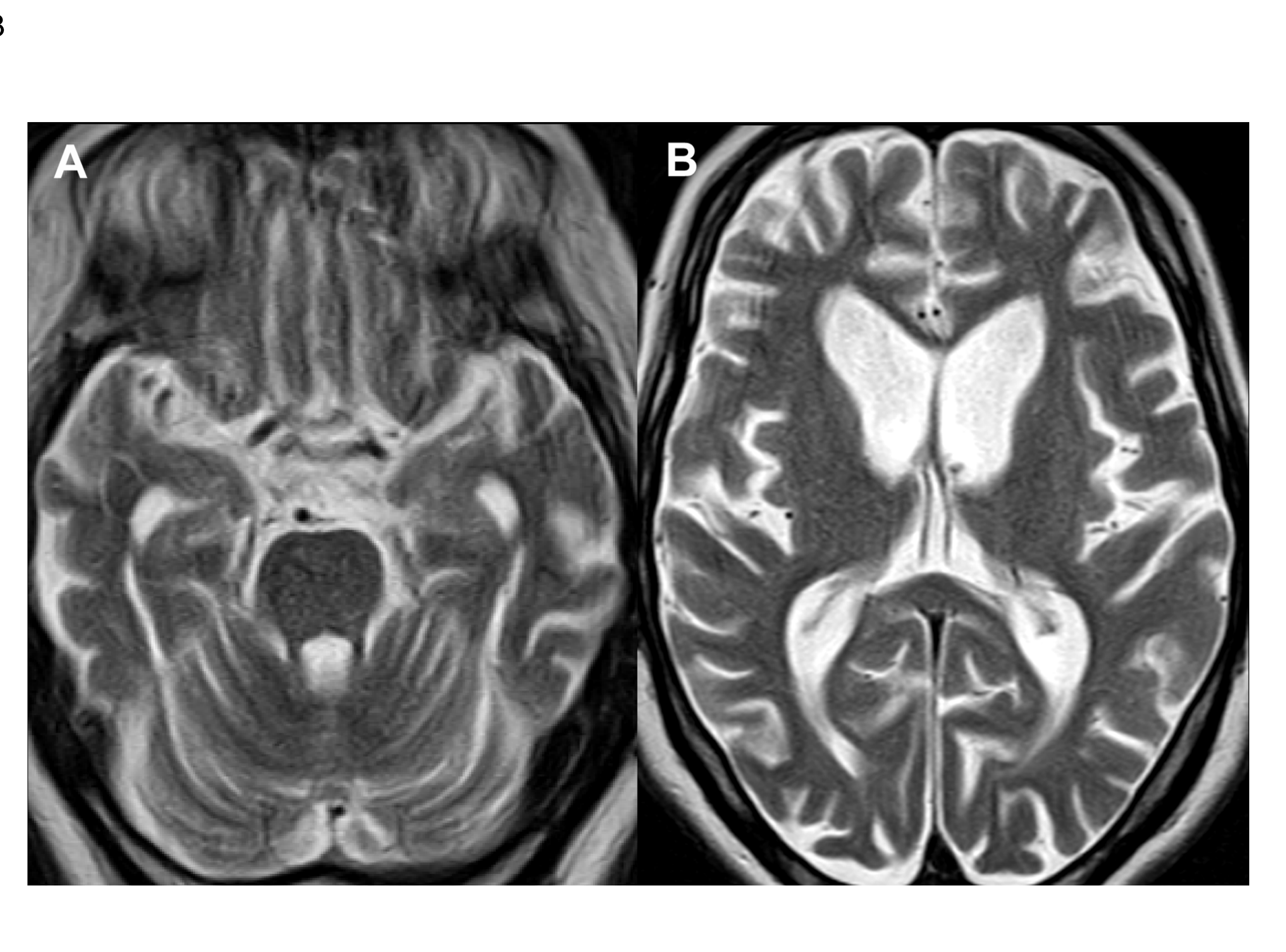

Fig. 3
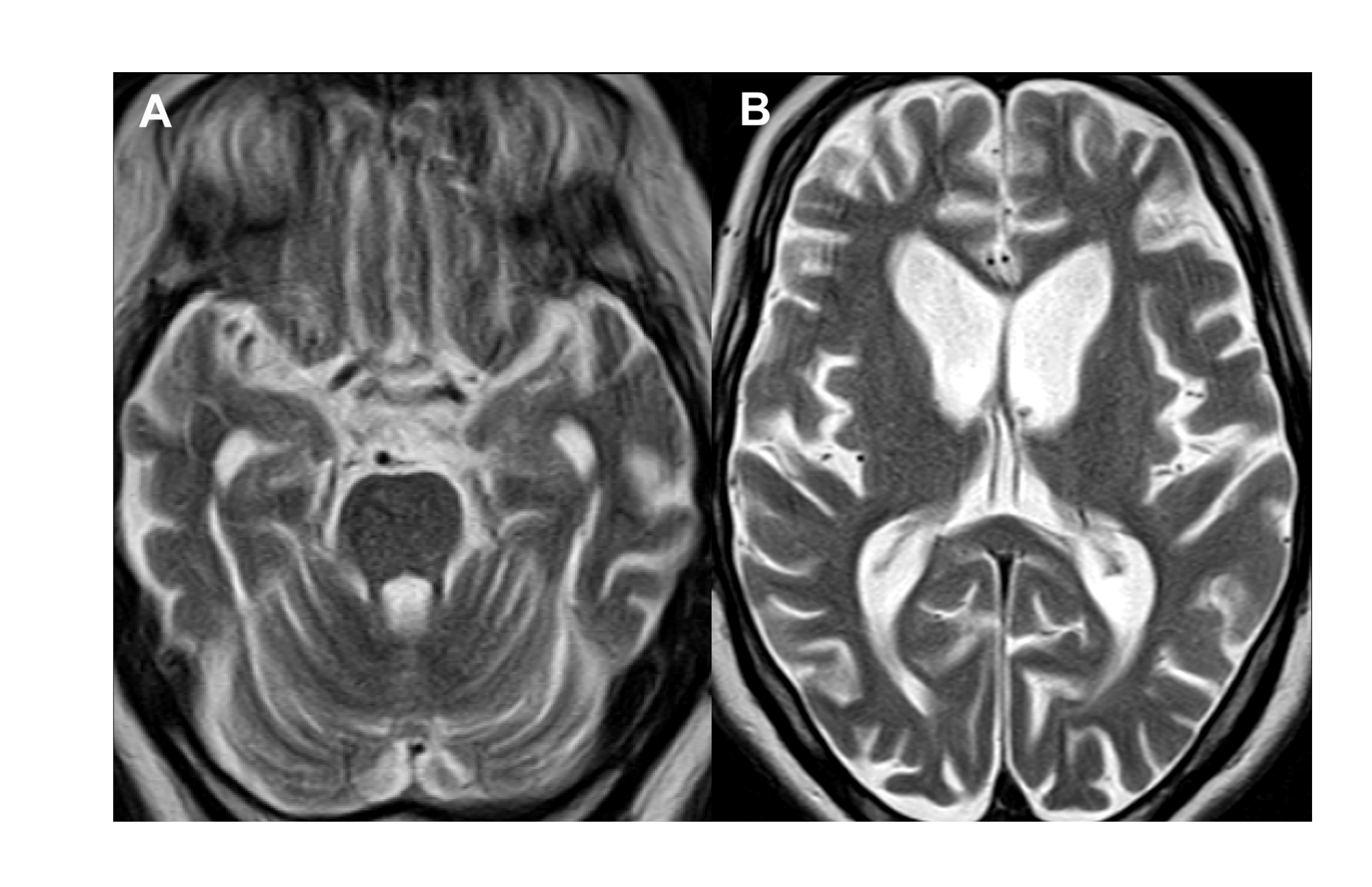